\title{
Determinação do conteúdo fenólico e avaliação da atividade antioxidante de Acacia podalyriifolia A. Cunn. ex G. Don, Leguminosae-mimosoideae
}

\author{
Cláudia Alexandra de Andrade ${ }^{1}$, Camila Klocker Costa ${ }^{1}$, Karina Bora ${ }^{1}$, Marilis Dallarmi \\ Miguel $^{2}$, Obdúlio Gomes Miguel ${ }^{1}$, Vitor Alberto Kerber ${ }^{1 *}$
}

\author{
${ }^{1}$ Laboratório de Fitoquímica, Departamento de Farmácia, Universidade Federal do Paraná, \\ Av. Prefeito Lothario Meissner, 3400, 80210-170, Curitiba, PR, Brasil, \\ ${ }^{2}$ Laboratório de Farmacotécnica, Departamento de Farmácia, Universidade Federal do Paraná, \\ Av. Prefeito Lothario Meissner, 3400, 80210-170, Curitiba, PR, Brasil
}

\begin{abstract}
RESUMO: Espécies de Acacia são tradicionalmente utilizadas para o tratamento de diversas patologias. Estudos quimiotaxonômicos têm relacionado o gênero Acacia como fonte de compostos fenólicos, os quais apresentam propriedades biológicas diversas. Este trabalho destaca a atividade antioxidante de Acacia podalyriifolia. Esta planta é originária da Austrália e amplamente cultivada no Sul do Brasil como ornamental. O material vegetal (flores) foi seco à sombra, submetido à extração com etanol, seguido de partição com hexano, diclorometano e acetato de etila. A triagem fitoquímica realizada no extrato etanólico bruto e nas suas frações, demonstrou a presença de compostos fenólicos e flavonóides. A determinação do conteúdo fenólico foi realizada com o reativo Folin Ciocalteau, verificando ser a fração acetato de etila (FAE) a que apresentou a maior concentração (338,5 mg de ácido gálico/g de FAE). Na avaliação da atividade captadora de radical, empregando o radical livre DPPH, a fração acetato de etila demonstrou atividade antioxidante mais elevada $\left(\mathrm{IC}_{50}=3,22 \mu \mathrm{g} / \mathrm{mL}\right.$ de FAE).
\end{abstract}

Unitermos: Acacia podalyriifolia, antioxidante, compostos fenólicos.

\begin{abstract}
Determination of the phenolic content and evaluation of the antioxidant activity of Acacia podalyriifolia A. Cunn. ex G. Don, Leguminosae-mimosoideae”. Acacia species are usually used as treatment for several diseases. Chemotaxonomic studies have related the Acacia genus as a source of phenolic compounds, which present several biological properties. This work emphasizes the antioxidant activity of Acacia podalyriifolia. This plant is natural from Australia and vastly cultivated in the South of Brazil as ornamental feature. The plant material (flowers) was shadow-dried and submitted to the extraction with ethanol, followed by partition with hexane, dichloromethane and ethyl acetate. The phytochemical screening performed in the ethanol crude extract and in its fractions showed the presence of phenolic compounds and flavonoids. Determination of the phenolic content was performed with the Folin Ciocalteau reagent. It was found out that the ethyl acetate fraction (FAE) was the one that displayed the biggest concentration (338,5 $\mathrm{mg}$ of gallic acid/g of FAE). In the evaluation of the radical scavenging activity, using the free radical DPPH, the ethyl acetate fraction showed the highest antioxidant activity $\left(\mathrm{IC}_{50}=3,22\right.$ $\mu \mathrm{g} / \mathrm{mL}$ from FAE).
\end{abstract}

Keywords: Acacia podalyriifolia, antioxidant, phenolic compounds.

\section{INTRODUÇÃO}

O oxigênio molecular e seus radicais são os reagentes mais importantes na bioquímica dos radicais livres nas células aeróbicas. O termo "espécies reativas de oxigênio" (ERO) inclui os radicais livres contendo oxigênio, como o ânion superóxido $\left(\mathrm{O}_{2}-\right)$, o radical hidroxila (HO.), o radical peroxila (ROO.) e espécies não radicalares como o peróxido de hidrogênio $\left(\mathrm{H}_{2} \mathrm{O}_{2}\right)$ e o oxigênio singlete $\left({ }^{1} \mathrm{O}_{2}\right)$, os quais são frequentemente gerados como subprodutos de reações biológicas ou por fatores exógenos (Gyamfi et al.,1999; Gülcin et al., 2003).

Estas espécies reativas de oxigênio podem causar um grande número de desordens celulares ao reagir com lipídeos, proteínas, carboidratos e ácidos nucléicos. Estas espécies estão envolvidas tanto no processo de envelhecimento, como também em muitas complicações biológicas, incluindo inflamação crônica, problemas respiratórios, doenças neurodegenerativas, Diabetes mellitus, aterosclerose, doenças auto-imunes das glândulas endócrinas, carcinogênese e mutagênese 
(Gyamfi et al., 1999; Al-Mamary et al., 2002; Gülcin et al., 2003; Chanwitheesuk et al., 2005).

Antioxidantes são substâncias que retardam ou previnem significativamente a oxidação de lipídios ou outras moléculas ao inibirem a iniciação ou a propagação da reação de oxidação em cadeia (Al-Mamary et al., 2002; Moreira et al., 2002; Chanwitheesuk et al., 2005; Wu et al., 2005; Lima et al., 2006), além de prevenirem ou repararem danos ocasionados às células pelas espécies reativas de oxigênio (Chanwitheesuk et al., 2005).

As substâncias com núcleo fenólico, como tocoferol, flavonóides e ácidos fenólicos, apresentam destaque especial como antioxidantes, por atuarem como eficientes captadores de espécies reativas de oxigênio (EROs), além de reduzirem e quelarem íons férrico que catalisam a peroxidação lipídica (Al-Mamary et al., 2002; Nahar; Sarker, 2005; Delazar et al., 2006).

Estudos quimiotaxonômicos relacionam o gênero Acacia como reconhecida fonte de substâncias fenólicas (Kerber; Silva, 1993; Andrade et al., 2003; Pansera et al., 2003), os quais apresentam uma grande variedade de ações biológicas (Duroux et al., 1998; Peitz et al., 2003), dentre elas a atividade antioxidante.

Estudos etnobotânicos revelaram que diferentes espécies de Acacia têm sido empregadas no tratamento das mais diversas patologias (Hagos; Samuelsson, 1988; Chhabra et al., 1990; Nabi et al., 1992; Sekine et al., 1997; Kambizi; Afolayan, 2001; Wu et al., 2005).

Ação antioxidante foi observada em diferentes espécies do gênero Acacia. Extratos de A. catechu (Park; Boo, 1991; Naik et al., 2003), A. confusa (Chang et al., 2001; Wu et al., 2005); A. nilotica (Saleem et al., 2001) e A. pennata (Thalang et al., 2001) apresentavam efeitos antioxidantes devido à presença de substâncias polifenólicas, como flavonóides e taninos. Mel obtido de A. ehrenbergina (Al-Mamary et al., 2002) e de outras espécies de Acacia (Meda et al., 2005), apresentou elevado potencial antioxidante e teor de compostos fenólicos. Extratos e compostos flavonoídicos obtidos de A. mangium e A. auriculiformis também demonstraram intensa atividade captadora de radicais livres (Mihara et al., 2005).

Também conhecida como Acacia-minosa, Acacia podalyriifolia A. Cunn. ex G. Don, LeguminosaeMimosoideae, é uma planta exótica, originária da Austrália, amplamente difundida no sul do Brasil pelo cultivo como árvore ornamental (Burkart, 1979; Duarte; Wolf, 2005).

Os trabalhos fitoquímicos descritos para Acacia podalyriifolia foram realizados com as folhas, goma e flores desta espécie. Alcalóides foram obtidos por White (1943) e por Ballandrin et al. (1978) a partir do extrato etanólico das folhas. Polissacarídeos da goma foram analisados por Anderson \& Bell (1976) e por Churms et al. (1977). Substâncias fenólicas flavonoídicas foram obtidas do fracionamento do extrato etanólico das flores por Andrade et al. (2005).
O interesse no potencial antioxidante de Acacia podalyriifolia foi reforçado pela presença de substâncias fenólicas flavonoídicas nas suas flores e por não haver relato na literatura destes estudos para esta espécie (Andrade et al., 2005).

As metodologias mais comuns para se determinar a atividade antioxidante de modo prático, rápido e sensível são as que envolvem um radical cromóforo, simulando as espécies reativas de oxigênio (EROs), sendo o radical livre DPPH (1,1-difenil-2picrilhidrazina) um dos mais utilizados (Arnao et al., 2000). O radical livre DPPH é um cromóforo muito estável, com um pico de absorção no comprimento de onda de $517 \mathrm{~nm}$, em meio etanólico, apresentando solução de coloração violeta intensa (Blois; 1958; Arnao et al., 2000). À medida que o DPPH sofre redução pelos componentes presentes na solução teste, observa-se mudança da coloração da solução original de violeta intensa para amarela, proporcional à concentração da substância com potencial antioxidante presente, em conformidade com as leis de Lambert e Beer, podendo ser medida espectrometricamente a $517 \mathrm{~nm}$ (Blois, 1958).

\section{MATERIAL E MÉTODOS}

\section{Material vegetal}

Flores de A. podalyriifolia foram coletadas em Curitiba, entre os meses de junho a setembro de 2004. O material foi identificado pelo botânico Gert Hatschbach do Museu Botânico Municipal de Curitiba e sua exsicata encontra-se depositada no mesmo local sob o número 268.219.

\section{Preparo dos extratos vegetais}

Após secagem à sombra, flores de $A$. podalyriifolia foram submetidas à extração com etanol, seguida de partição líquido-líquido com hexano, diclorometano e acetato de etila, segundo metodologia descrita por Kerber \& Silva (1993) e Andrade et al. (2005), obtendo-se o extrato etanólico bruto (EEB), as frações diclorometano (FDCM) e acetato de etila (FAE). Alíquotas de EEB, FDCM e FAE foram conduzidas à secura, em evaporador rotatório sob pressão reduzida e então dissolvidas em etanol, para serem utilizadas na determinação do conteúdo de compostos fenólicos e nos testes de atividade antioxidante.

\section{Determinação do conteúdo de compostos fenólicos}

O conteúdo de compostos fenólicos no extrato etanólico bruto (EEB) e nas frações diclorometano (FDCM) e acetato de etila (FAE) foi realizado baseado no método colorimétrico de Folin-Ciocalteau (Kujala et al., 2000; Wu et al., 2005; Meda et al., 2005), com algumas modificações. 
Alíquotas de EEB, FDCM e FAE foram diluídas com água destilada para se obter concentrações de 25 a $125 \mu \mathrm{g} / \mathrm{mL}$. A $0,5 \mathrm{~mL}$ de cada amostra foram adicionados $0,5 \mathrm{~mL}$ do reagente de Folin-Ciocalteau $2 \mathrm{~N}$ e 1,0 mL de água. Após um período de 2 a 5 minutos, foi acrescentado aos tubos $0,5 \mathrm{~mL}$ de carbonato de sódio $\left(\mathrm{Na}_{2} \mathrm{CO}_{3}\right)$ a $10 \%$. Após 1 hora de incubação à temperatura ambiente, a absorbância foi mensurada em espectrofotômetro a 760 $\mathrm{nm}$, usando água destilada como branco. Ácido gálico (2,5 a $12,5 \mu \mathrm{g} / \mathrm{mL})$, dissolvido em água destilada, foi usado para elaboração da curva de concentração padrão e os valores de fenólicos totais foram expressos como equivalentes de ácido gálico (mg de ácido gálico/g de amostra).

\section{Avaliação da atividade antioxidante}

A ação antioxidante da Acacia podalyriifolia foi analisada pela capacidade dos antioxidantes presentes na amostra captarem o radical livre DPPH (1,1-difenil2-picrilhidrazina), conforme as metodologias descritas por Gyamfi et al. (1999), Meda et al. (2005) e Wu et al. (2005), com algumas modificações.

Para a avaliação da atividade captadora do radical livre DPPH (1,1-difenil-2-picrilhidrazina), alíquotas de EEB, FDCM e FAE (amostras), ácido ascórbico e rutina (padrões) foram diluídas com etanol para se obter as concentrações de 0,5 a $100 \mu \mathrm{g} / \mathrm{mL}$. A 3 $\mathrm{mL}$ de cada amostra foi acrescentado $0,1 \mathrm{~mL}$ de solução etanólica do radical livre DPPH 1 mM. Após 30 minutos de incubação à temperatura ambiente, ao abrigo da luz, a redução do radical livre DPPH foi mensurada pela leitura da absorbância em 517 nm, contra um branco específico em cada avaliação, formado somente pelas amostras nas suas respectivas diluições. Como controle, foi utilizado $0,1 \mathrm{~mL}$ de solução etanólica de DPPH $1 \mathrm{mM}$ e $3 \mathrm{~mL}$ de etanol.

Para avaliar a atividade captadora de radical, foi obtida a porcentagem de inibição, conforme a equação:

\% de Inibição = [(Absorbância do controle - absorbância da amostra)/absorbância do controle] X 100

A determinação da $\mathrm{IC}_{50}$, ou seja, concentração da amostra ou padrão que causa $50 \%$ de inibição da concentração inicial de DPPH, foi obtida por regressão linear dos pontos plotados graficamente. Para a plotagem dos pontos, foram utilizados os valores das médias obtidas de triplicatas realizadas para cada um dos testes. Para detectar as diferenças entre as médias e avaliar estas diferenças foi utilizada análise de variância (ANOVA) e o teste de Tukey com 95\% de nível de confiança.

\section{RESULTADOS}

\section{Determinação do conteúdo de compostos fenólicos}

O conteúdo de compostos fenólicos (mg de ácido gálico/g de amostra) foi de 206,4 para o extrato etanólico bruto (EEB), 240,2 para a fração diclorometano (FDCM) e 338,5 para a fração acetato de etila (FAE), usando a curva padrão do ácido gálico $\left(\mathrm{R}^{2}=0,9945\right)$.

\section{Avaliação da atividade antioxidante}

A porcentagem de inibição do radical DPPH (\% inibição), para as amostras e padrões (0,50 a $100 \mu \mathrm{g} /$ $\mathrm{mL}$ ) está representada no Gráfico 1 . Os valores médios de concentração das amostras (EEB, FDCM e FAE) e dos padrões (ácido ascórbico e rutina), que causam 50\% de inibição do radical DPPH $\left(\mathrm{IC}_{50}\right)$ estão representados na Tabela 1. Os dados foram submetidos à análise de variância (ANOVA) e ao teste de Tukey, sendo $p<0,05$ considerado significativo. O valor de $\mathrm{IC}_{50}$ de $3,22 \mu \mathrm{g} / \mathrm{mL}$ obtido para a fração acetato de etila foi significativamente diferente $(p<0,05)$ dos obtidos para o padrão de ácido ascórbico $(0,49)$, para o extrato etanólico bruto $(10,48)$ e para a fração diclorometano $(36,65)$, mas não difere estatisticamente $(p>0,05)$ do apresentado pelo padrão de rutina $(4,14)$.

\section{DISCUSSÃO}

Muitas substâncias naturais, obtidas das plantas, têm sido identificadas como captadoras de espécies reativas de oxigênio, protegendo o corpo humano dos efeitos destes, bem como retardando o aparecimento de muitas doenças crônicas (Gülcin et al., 2003).

As substâncias com núcleo fenólico apresentam destaque especial como eficientes captadores radicalares, como demonstrado por Gyamfi et al. (1999), Kujala et al. (2000), Chang et al. (2001), Sallem et al. (2001), Thalang et al. (2001), Al-Mamary et al. (2002); Mihara et al. (2005); Meda et al. (2005); Wu et al. (2005).

O gênero Acacia é reconhecido como fonte de compostos fenólicos, sendo que a espécie objeto deste estudo, Acacia podalyriifolia, apresentou considerável quantidade desta categoria de substâncias, encontrados com maior concentração na fração acetato de etila.

A atividade antioxidante foi avaliada pela determinação da captação do radical DPPH $\left(\mathrm{IC}_{50}\right)$ pelo extrato etanólico bruto, pelas frações diclorometano e acetato de etila e pelos padrões de ácido ascórbico e rutina. O padrão de ácido ascórbico demonstrou ser significativamente mais ativo que os demais. A fração acetato de etila apresentou atividade antioxidante significativamente maior que o extrato etanólico bruto e a fração diclorometano. Comparada ao padrão de rutina, a fração acetato de etila apresentou atividade similar, não sendo significativa a diferença nos valores de $\mathrm{IC}_{50}$ obtidos.

Os resultados obtidos para a Acacia podalyriifolia confirmam o que já havia sido verificado com A. catechu, estudos realizados por Park; Boo (1991) 


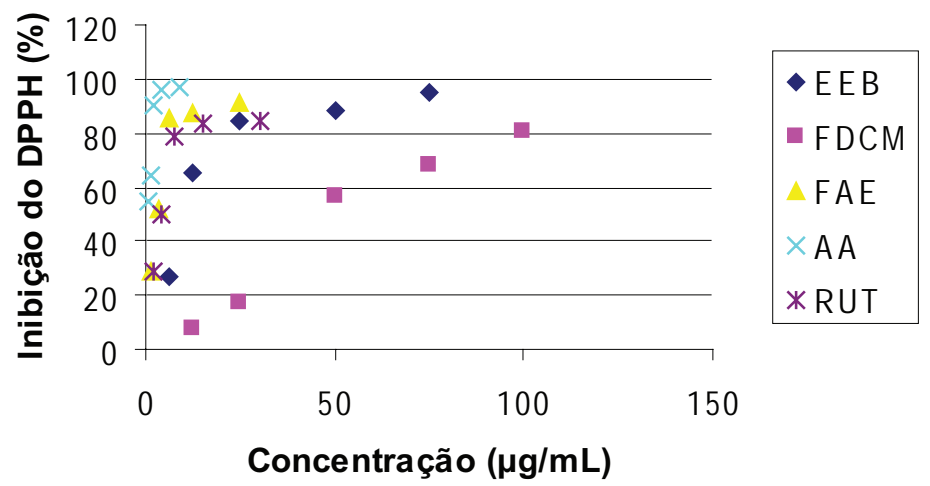

EEB: extrato etanólico bruto; FDCM: fração diclorometano; FAE: fração acetato de etila; AA: ácido ascórbico; RUT: rutina

Gráfico 1. Porcentagem de inibição do radical DPPH pelas amostras (EEB, FDCM e FAE) e pelos padrões (ácido ascórbico e rutina).

Tabela 1. Atividade captadora do radical DPPH (IC50).

\begin{tabular}{l|c}
\hline Amostras/Padrões & $\mathrm{IC}_{50}(\mu \mathrm{g} / \mathrm{mL})$ \\
\hline Ácido ascórbico & 0,49 \\
Rutina & 4,14 \\
Extrato etanólico bruto & 10,48 \\
Fração diclorometano & 36,65 \\
Fração acetato de etila & 3,22 \\
\hline
\end{tabular}

$\mathrm{IC}_{50}=$ Concentração que inibe $50 \%$ da concentração inicial do radical DPPH

e Naik et al. (2003); A. confusa por Chang et al. (2001) e Wu et al. (2005); A. nilotica por Sallem et al. (2001); $A$. pennata por Thalang et al. (2001); mel obtido de flores de espécies diversas de Acacia por Al-Mamary et al. (2002) e Meda et al. (2005); A. mangium e A. auriculiformis por Mihara et al. (2005), que apresentaram efeitos antioxidantes importantes devido ao teor de substâncias fenólicas.

\section{CONCLUSÃO}

Os estudos realizados com o extrato etanólico bruto e as frações diclorometano e acetato de etila, obtidos das flores de Acacia podalyriifolia, demonstraram que o conteúdo de compostos fenólicos foi mais elevado na fração acetato de etila, o que explicaria a maior ação antioxidante, demonstrada pela atividade captadora de radical $\left(\mathrm{IC}_{50}\right)$, ter sido observada com esta amostra.

\section{AGRADECIMENTOS}

Os autores agradecem ao botânico Gert Hatschbach, do Museu Botânico Municipal de Curitiba (MBM), pela identificação da espécie vegetal e ao estatístico Jorge Pontes pela análise estatística dos dados.

\section{REFERÊNCIAS}

Al-Mamary M, Al-Meeri A, Al-Habori M 2002. Antioxidant activities and total phenolics of different types of Honey. Nutr Res 22: 1041-1047.

Anderson DMW, Bell PC 1976. Partial structural studies of four Acacia gums exudates of the series Phyllodineae. Phytochemistry 15: 301-303.

Andrade CA, Silva VC, Peitz C, Miguel MD, Miguel OG, Kerber VA 2003. Revisão do gênero Acacia - atividades biológicas e presença de fenóis derivados do núcleo flavânico. Visão Acadêmica 4: 47-56.

Andrade CA, Peitz C, Cunico M, Carvalho JLS, Abrahao WM, Miguel MD, Miguel OG, Kerber VA 2005. Avaliação da atividade antibacteriana e triagem fitoquímica das flores de Acacia podalyriifolia A. Cunn. ex. G. Don Leguminosae-Mimosoidea. Rev Bras Farmacogn 15: 13-15.

Arnao MB, Cano A, Acosta M 2000. A method to measure antioxidant activity in organic media: application to lipophilic vitamins. Redox Rep 5: 365-370.

Balandrin MF, Kinghorn AD, Smolenski SJ, Dobberstein RH 1978. Reverse-phase High-Pressure Liquid Chromatography of some tryptamine derivatives. $J$ Chromatogr 157: 365-370.

Blois MS 1958. Antioxidant determination by the use of a stable free radical. Nature 181: 1199-1200.

Burkart A 1979. Leguminosas - Mimosoideas. In: Flora Ilustrada Catarinense. Itajaí: P. Raulino Reitz, p.1748. 
Chang ST, Wu JH, Wang SY, Kang PL, Yang NS, Shyur LF 2001. Antioxidant activity of extracts from Acacia confusa bark and heartwood. J Agric Food Chem 49: 3420-3424.

Chanwitheesuk A, Teerawutgulrag A, Rakariyatham N 2005. Screening of antioxidant activity and antioxidant compounds of some edible plants of Thailand. Food Chem 92: 491-497.

Chhabra SC, Uiso FC 1990. A survey of medicine plants of Eastern Tanzania for alkaloids, flavonoids, saponins and tannins. Fitoterapia 4: 307-316.

Churms SC, Merrifield EH, Stephen AM 1977. Structural features of the gum exudates from some Acacia species of the series Phyllodineae and Botrycephalae. Carbohyd Res 55: 3-10.

Delazar A, Talischi B, Nazemiyeh H, Rezazadeh H, Nahar L, Sarker SD 2006. Chrozophorin: a new acylated flavone glucoside from Chrozophora tinctoria (Euphorbiaceae). Rev Bras Farmacogn 16: 286-290.

Duarte MR, Wolf S 2005. Anatomical characters of the phyllode and stem of Acacia podalyriifolia A. Cunn. ex G. Don (Fabaceae). Rev Bras Farmacogn 15: 71-76.

Duroux L, Delmotte FM, Lancelin JM, Keravis G, Allemand C 1998. Insight into naphthoquinone metabolism: betaglucosidase-catalysed hydrolysis of hydrojuglone beta-D-glucopyranoside. Biochem J 333: 275-283.

Gülcin I, Oktay M, Kirecci E, Küfrevioglu OI 2003. Screening of antioxidant and antimicrobial activities of anise (Pimpinella anisun L) seed extracts. Food Chem 83: 371-382.

Gyamfi MA, Yonamine M, Aniya Y 1999. Free-radical scavenging action of medicinal herbs from Ghana Thonningia sanguinea on experimentally-induced liver injuries. Gen Pharmacol 32: 661-667.

Hagos M, Samuelsson G 1988. Quantitative determination of quracol A, B and (+)-fisetinidol in bark and gum of Acacia tortilis. Acta Pharm Suec 25: 321-324.

Kambizi L, Afolayan AJ 2001. An ethnobotanical study of plants for the treatment of sexually transmitted disease (njovera) in Guruve District, Zimbabwe. $J$ Ethnopharmacol 77: 5-9.

Kerber VA, Silva GAAB 1993. Flavonóides da Acacia longifolia (Andr.) Willd. - Leguminosae-Mimosoideae. Rev Bras Farm 74: 16-18.

Kujala TS, Loponen JM, Klika KD, Pihlaja K 2000. Phenolics and betacyanins in red beetroot (Beta vulgaris) root: distribuition and effect of cold storage on the content of total phenolics and three individual compounds. $J$ Agric Food Chem 48: 5338-5342.

Lima AR, Barbosa VC, Santos Filho PR, Gouvêa CMCP 2006. Avaliação in vitro da atividade antioxidante do extrato hidroalcoólico de folhas de bardana. Rev Bras Farmacogn 16: 531-536.

Meda A, Lamien CE, Romito M, Millogo J, Nacoulma OG 2005. Determination of the total phenolic, flavonoid and proline contents in Burkina Fasan honey, as well as their radical scavenging activity. Food Chem 91:
571-577.

Mihara R, Barry KM, Mohammed CL, Mitsunaga T 2005. Comparison of antifungal and antioxidant activities of Acacia mangium and Acacia auriculiformis heartwood extracts. J Chem Ecol 31: 789-804.

Moreira DL, Engelhardt RL, Reis AS, Sanches EM, Leitão SG, Leitão GG 2002. Substâncias fenólicas com atividade antioxidante de Pseudopiptadenia contorta (Leguminosae-Mimosoideae). Rev Bras Farmacogn 12(Supl.): 124-125.

Nabi ABD, Reisinger EC, Reinthaler FF, Still F, Eibel U, Krejs GJ 1992. Antimicrobial activity of Acacia nilotica (L) Willd ex Del var. nilotica (Mimosaceae). J Ethnopharmacol 37: 77-79.

Nahar L, Sarker SD 2005. Chenoalbuside: an antioxidant phenolic glycoside from the seeds of Chenopodium album L. (Chenopodiaceae). Rev Bras Farmacogn 15: 279-282.

Naik GH, Priyadarsinik K, Satau JG, Banavalikar MM, Sohoni DP, Biyani M, Mohan H 2003. Comparative antioxidant activity of individual herbal components used in Ayurvedic medicine. Phytochemistry 63: 97104.

Peitz C, Cúnico MM, Miguel OG, Miguel MD, Kerber VA 2003. Avaliação da atividade antibacteriana e triagem fitoquímica das folhas de Acacia longifolia (Andr.) Willd. (Leguminosae). Rev Bras Farmacogn 13: 6165.

Pansera MR, Santos ACA, Paese K, Wasum R, Rossato M, Rota LD, Pauletti GF, Serafini LA 2003. Análise de taninos totais em plantas aromáticas e medicinais cultivadas no Nordeste do Rio Grande do Sul. Rev Bras Farmacogn 13: 17-22.

Park SN, Boo YC 1991. Flavonoids for protection of cells against chemically active species of oxygen, their extraction from plants, and their use in cosmetics. $\mathrm{Fr}$ Demande $17 \mathrm{p}$.

Saleem A, Ahotupa M, Pihlaya K 2001. Total phenolics concentration and antioxidant potential of extracts of medicinal plants of Pakistan. Z Naturforsch 56c: 973978.

Sekine T, Fukasawa N, Ikegami F, Saito K, Fujii Y, Murakoshi I 1997. Structure and synthesis of a new monoterpenoidal carboxiamide from the seeds of the Thai medicinal plant Acacia concinna. Chem Pharm Bull 45: 148-151.

Thalang VN, Trakoontivakorn G, Nakaharak K, Gassinee $\mathrm{T}$ 2001. Antioxidant activity of some commonly consumed leafty vegetables in Thailand. Jircas $J$ 9: 35-43.

White EP 1943. Alkaloids of the Leguminosae. J Sci Tech 25: 93-114.

Wu JH, Tung YT, Wang SY, Shyur LF, Kuo YH, Chang ST 2005. Phenolic antioxidants from the heartwood of Acacia confusa. J Agric Food Chem 53: 5917-5921. 